\title{
MEMS-GC with Integrated Heater for Ion Trap Mass Spectrometer
}

\author{
Tzu-Hsuan Chang ${ }^{1}$, Daniel Struk ${ }^{1}$, Milad Navaei ${ }^{1}$, Vladimir M. Doroshenko ${ }^{2}$; Victor Laiko ${ }^{2}$; Eugene \\ Moskovets'; Konstantin Novoselov', Jean-Marie D. Dimandja ${ }^{1}$ Peter J. Hesketh ${ }^{1}$, \\ ${ }^{1}$ School of Mechanical Engineering, Georgia Institute of Technology, Atlanta GA 30332, USA, \\ ${ }^{2}$ MassTech Inc., Columbia, MD 21046, USA \\ peter.hesketh@me.gatech.edu
}

\begin{abstract}
:
In this work a microfabricated 6 meter long gas chromatography column has been integrated with a platinum resistive heater and installed in an ion-trap mass spectrometer. The system is for the detection and identification of volatile organic compounds. The system was demonstrated to separate a mixture of sixteen volatile organic compounds with a maximum temperature of $150{ }^{\circ} \mathrm{C}$.
\end{abstract}

Key words: MEMS, GC-MC, volatile organic compounds, gas chromatography.

\section{Introduction}

The detection and analysis of volatile organic compounds has long been a challenge in portable instrumentation because of the ability to deal with a complex background mixture. Applications include environmental sensing, breath analysis and detection of species for first responders to assess risks in a given situation, for example before entering a burning building.

\section{Background}

Gas chromatography in a miniature format has been explored for many years (1) and has been reviewed recently (2). The MEMS column can be combined with miniature sensors, such as thermal conductivity to realize compact portable vapor analysis systems (3) or combined with nanomechanical resonators to realize mass analysis of compounds eluted from the column (4). Other developments include the use of short columns for fast analysis (5) and temperature zone programing to improve separation performance (6).

\section{Approach}

This is the first time a MEMS-GC has been integrated into a mass spectrometer. The ion trap mass spectrometer is compact and operates at low gas flow rates which make it a particularly good match for MEMS GC separation columns which have small dimensions. The mass spectrometer provides species identification which can be combined with the retention time data from the GC separation to increase confidence of chemical identification from a sample being analyzed.

\section{Column Fabrication}

The MEMS-GC columns were fabricated in silicon. First, the ports serving for gas inlet with $200 \mu \mathrm{m}$ in circumference were lithographically patterned on the back side of the wafer. Next, a deep reactive-ion-etch process was used to etch $250 \mu \mathrm{m}$ deep holes halfway through silicon wafer anisotropically. Lithography and etching was repeated on the topside of the wafer for the spiral channel $40 \mu \mathrm{m}$ in width, illustrated as Fig. 1(a). A second double sided polished 4 inch silicon wafer was used to seal the column. A thin layer of gold ( $\mathrm{Cr} / \mathrm{Au}, 20 / 100 \mathrm{~nm}$ ) was deposited on the one side of wafer for eutectic bonding process. The bonded die was then annealed at $1100{ }^{\circ} \mathrm{C}$ for two hours to ensure gold atoms diffuse into the silicon and form a gold silicon alloy in order to enhance the bonding strength. Finally, a 20/100 nm thick $\mathrm{Ti} / \mathrm{Pt}$ layer was deposited using e-beam deposition and lift-off process to form the resistive heater on the surface, illustrated as Fig. 1(c).

(a)

(b)

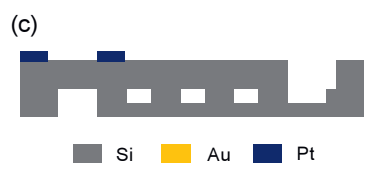

Fig. 1. Illustration of micro-fabricated GC column, (a) the channel substrate with gas inlet, (b) the lid and gas outlet, (c) the final device with Pt resistive heater after bonding. 
Fig. 2 shows SEM images of micro-fabricated GC-column. Fig. 2(a) was taken immediately after channel etching. Fig. 2(b) shows the side view of bonded chip, indicated the robustness of bonding interface.

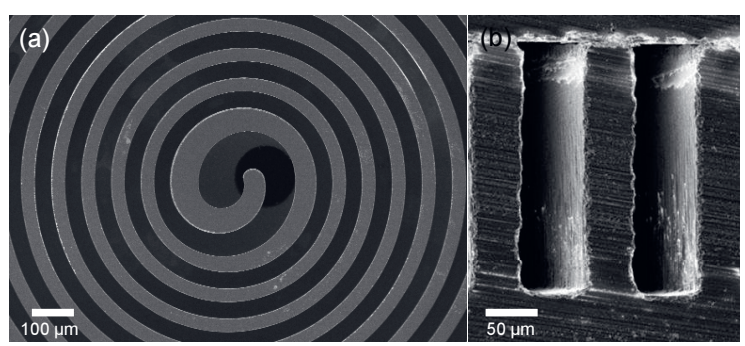

Fig. 2. SEM images of micro-fabricated GC column, (a) top view, (b) side view after bonding with lid.

\section{Stationary Phase Coating}

A solution of the stationary phase, OV-1 $5 \mathrm{mg} / \mathrm{ml}$, was prepared in a $50 \%$ by volume mixture of pentane and dichloromethane with $1 \%$ by weight dicumyl peroxide as a crosslinking agent and then introduced into the column under static coating conditions for 2 hours. The liquid was evacuated with a vacuum pump and the column was annealed in dry gas, helium for 4 hours at $180{ }^{\circ} \mathrm{C}$. After coating, the column was tested with a mixture of volatile organic compounds and the effect of different temperature ramps was examined on the separation performance and analysis time.

\section{Results and Discussion}

The PDMS/DVB SPME was found to give the best results with a 1-minute sampling time, sampling the headspace of a vial containing a mixture of sixteen compounds. Figure 3 shows the separation obtained with helium carrier gas using an initial temperature of $40^{\circ} \mathrm{C}$ held constant for 2 minutes then ramped at $5{ }^{\circ} \mathrm{C} / \mathrm{min}$ to $150{ }^{\circ} \mathrm{C}$. Separation of all the compounds is observed in under 45 minutes.

\section{Conclusions}

This work demonstrates a compact integrated MEMS-GC with ion-trap MS system which is capable of separation compounds in a mixture from a SPME or a liquid injection sample.

\section{Acknowledgments}

We gratefully acknowledge the financial support for project MAGELIN from iARPA and the technical advice from colleagues at the Institute of Electronics and Nanotechnology at Georgia Tech.

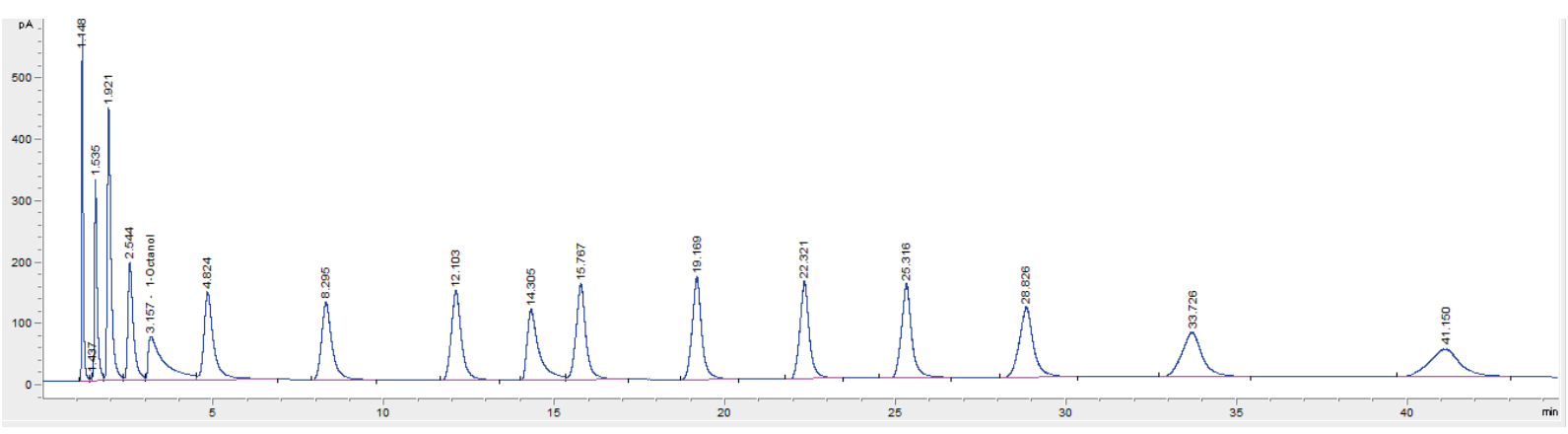

Fig. 3. Chromatograph for 50 um wide, 250 um deep channel of $6 \mathrm{~m}$ length coated with OV-1 using helium carrier gas at a flow rate of $10 \mathrm{~cm} / \mathrm{s}$ and temperature held constant at $40 \mathrm{C}$ for 2 minutes then ramped $5 \mathrm{C} / \mathrm{min}$ from 40 to 150 C. Sample was introduced with a tip split injection, a 16-component mixture of $C_{5}$ to $C_{17}$, benzene, pyridine, and 1-octanol.

\section{References}

[1] S. C. Terry, J. H. Jerman, J. B. Angell, IEEE Transact. Electron Dec., 26, 1880 (1979).

[2] F. Haghighi, Z. Palebpour, A. Sanati-Nexhad, Lab on a Chip, 15, 2559 (2015).

[3] A. Mahdavifar, M. Navaei, P. J. Hesketh, J. D. Dimandja, J. R. Stetter, G. McMurray, Journal of Solid State Science and Technology, 4, S1 (2015).

[4] A. Venkatasubramanian, V. T. K. Sauer, S. K. Roy, M. Xia, D. S. Wishart, W. K. Hiebert, Nano Letters, 16, 6975 (2016).

[5] J. Bryant-Genevier, E. T. Zellers, J. Chromatography A, 1422, 299 (2015).
[6] Z. Lin, N. Nunovero, J. Wang, R. Nidetz, S. Buggavetti, K. Kurabayashi, E. T. Zellers, Sensors and Actuators B, 254, 561 (2018). 